\title{
Mensajería instantánea y construcción compartida de significados: una experiencia de aprendizaje colaborativo en el Prácticum de Maestro de Educación Primaria
}

\section{Instant messaging and shared construction of meaning: An experience of collaborative learning in the Practicum of Primary Education Teacher}

\author{
José Manuel Martín Fernández \\ Universidad de Granada. España \\ jmmartinf@yahoo.es \\ Carmen Trigueros Cervantes \\ Universidad de Granada. España \\ ctriguer@ugr.es
}

\begin{abstract}
Resumen
La investigación sobre la relación entre las TIC y el aprendizaje colaborativo está en amplio desarrollo en los últimos años, sin embargo no se incide en el uso de la mensajería instantánea como herramienta para fomentar esta construcción compartida del aprendizaje desde un enfoque descriptivo, relacional e interpretativo. En este artículo presentamos los resultados referentes a una investigación en la que nos centramos en evaluar la mensajería instantánea como recurso para facilitar el aprendizaje colaborativo, favoreciendo la participación y comunicación que tiene lugar entre los estudiantes, los tutores y los supervisores del Prácticum II. Concretamente, utilizando una metodología cualitativa, analizamos la construcción compartida de significados como mecanismo interpsicológico del aprendizaje colaborativo realizado en los seminarios a través del chat. Los resultados muestran que dicho mecanismo se pone de manifiesto a través de los extensos debates generados a partir de los argumentos y discrepancias en las temáticas surgidas, la actitud solidaria de aporte de soluciones a problemas planteados, así como el planteamiento de cuestiones.
\end{abstract}

Palabras clave

Chat, Aprendizaje Colaborativo, Practicum, Educación Física.

\begin{abstract}
Recent years research on the relationship between ICT and collaborative learning is under extensive development, however it does not focus on the use of instant messaging as a tool to promote this shared construction of learning from descriptive, relational and interpretive approach. This paper is focused on the partial results of a research about the evaluation of instant messaging. The latter is used as a resource to foster collaborative learning by improving the involvement of students, professors and Practicum II supervisors. The building of shared meanings as social interaction mechanisms for collaborative learning that were carried out Online chat seminars is analysed by means of qualitative methodology. The results shown that these mechanisms are shown by long debates generated from the discussions and disagreements about the different themes arisen, by the manifestation of solidarity to contribute to find out solutions to suggested problems as well as by the raising of issues of concern.
\end{abstract}

\section{Keywords}

Chat, Collaborative Learning, Practicum, Physical Education. 


\section{Introducción.}

Las prácticas que el alumnado de la titulación de Maestro en Educación Primaria debe realizar en Centros Educativos para consolidar su formación como futuro profesional, en la Facultad de Ciencias de la Educación de Granada, se llevan a cabo en dos momentos. Un primer momento bajo la asignatura de $3^{\circ}$ curso Prácticum I, cuyo objetivo es aproximarse a la realidad educativa fundamentalmente desde la observación y reflexión; y un segundo momento en $4^{\circ}$ curso en la asignatura Prácticum II, en la que el estudiante debe efectuar actuaciones en el aula que le permitan integrar sus conocimientos teóricos con la práctica.

Sin embargo, la organización del Prácticum I y II de la Facultad de Ciencias de la Educación de Granada contempla una casuística, y es la de realizar dichas prácticas fuera de la provincia. Dado que la distancia implica que se puedan realizar pocos seminarios presenciales, en la presente investigación hemos recurrido al uso de las Tecnologías de la Información y la Comunicación (TIC) para la realización de los seminarios y tutorías dentro de la asignatura Prácticum II, lo que supone la virtualización de parte de dicha supervisión. Concretamente hemos optado por el uso de sesiones de mensajería instantánea (seminarios virtuales), ya que creemos que este tipo de herramientas que facilitan la comunicación entre personas independientemente de la distancia geográfica a la que se encuentren, favorecen espacios virtuales donde poder intercambiar reflexiones, debatir, resolver dudas, compartir experiencias. En consecuencia, como afirma Fernández Jiménez (2002), podemos generar conocimiento compartido y fomentar el aprendizaje colaborativo en el alumnado.

En este artículo presentamos los resultados, referentes a una investigación en la que nos centramos en evaluar la mensajería instantánea como recurso para facilitar el aprendizaje colaborativo, favoreciendo la participación y comunicación que tiene lugar entre los estudiantes del Prácticum II, los tutores (profesores de los Centro Educativos) y los supervisores (profesores de la Facultad). Concretamente analizamos la construcción compartida de significados como mecanismo interpsicológico del aprendizaje colaborativo realizado a través de entornos virtuales (Casanova, Álvarez y Gómez 2009), como son los seminarios a través del chat. Se trata pues de aprender a cooperar y cooperar para aprender.

\subsection{Aprendizaje colaborativo y TIC.}

En los últimos años encontramos diferentes estudios centrados en fomentar el aprendizaje colaborativo intentando destacar todas sus ventajas dentro del ámbito educativo (Cuadrado, Fernández, Monroy y Montaño, 2012; Gros, Guerra y Sánchez, 2005; Gutiérrez, Yuste, Cubo y Lucero, 2011; Roselli, 2011; Segarra, 2006); sin embargo, todavía nos encontramos con autores que afirman que en nuestro sistema educativo el clima competitivo, en el que el éxito de unos puede ocasionar la exclusión de otros, prima sobre una cultura de la colaboración (Fernández y Malvar, 1999; Imbernón, 2000; Lorente, 2006).

Es por ello que se necesita fomentar un aprendizaje desde el punto de vista de la colaboración, a través de herramientas, recursos, o espacios socializadores, donde prime el respeto, actitudes activas y participativas, así como una interdependencia positiva que

Mensajería instantánea y construcción compartida de significados: una experiencia de aprendizaje colaborativo en el Prácticum de Maestro de Educación Primaria.

José Manuel Martín Fernández y Carmen Trigueros Cervantes 
lleve a un enriquecimiento social y cognitivo mutuo (Calzadilla, 2002; Johnson y Johnson, 1999; López Alacid, 2008).

En el ámbito de la investigación sobre la relación entre las TIC y el aprendizaje colaborativo, surge la perspectiva CSCL( Computer Supported Collaborative Learning) como un campo de estudio independiente introducido por Koschman (1996) enfocado a investigar la educación en ambientes tecnológicamente enriquecidos. Esta perspectiva está interesada en determinar: a) cómo el aprendizaje colaborativo introducido por computadoras puede mejorar la interacción entre parejas y equipos de trabajo, b) cómo la colaboración, así como la tecnología facilitan el conocimiento compartido y distribuido (Pizá y Madueño, 2007).

La investigación en este campo está en amplio desarrollo en los últimos años y es particularmente heterogénea y compleja (Dillenbourg, 1999); los aspectos que intervienen en el aprendizaje colaborativo (el comportamiento del grupo, el compromiso, la concepción compartida del problema, la tarea, etc.), suponen un punto de partida para diferentes líneas de investigación que a su vez se ramifican en detalles particulares de cada una de ellas (Gros, 2007; Koschmann, 1996). Sin embargo la mayoría de las investigaciones, trabajos y experiencias realizadas hasta el momento acerca de la utilización de la mensajería instantánea en el ámbito de la Educación Superior, apuntan las ventajas e inconvenientes de éste frente a otros recursos con un enfoque principalmente estructural y estadístico. Se utilizan plataformas web o campus virtuales, pero no se incide en el uso de la mensajería instantánea como herramienta para fomentar el aprendizaje colaborativo desde un enfoque descriptivo, relacional e interpretativo a través del cual podamos profundizar en los fenómenos educativos y sociales.

\subsection{Construcción compartida de significados.}

Si nos centramos en los estudios que han analizado foros de discusión a través de internet, que han llevado a cabo estudiantes en el ámbito universitario y cuyo objetivo era promover la construcción colaborativa de significados, revelan que, a pesar de realizarse un fluido intercambio de información y opiniones, los estudiantes no entran en profundidad en los debates, conceptos, ideas. Éstos no llegan a negociar sobre su significado, y mucho menos construir colaborativamente nuevas ideas (Onrubia, Naranjo y Segués, 2009). Esto nos lleva a entender el lenguaje, a modo de sistema de comunicación utilizado en los foros, como una mera transferencia de informaciones entre personas. No obstante, según Mercer (2001), cada vez que dialogamos con una persona participamos en un proceso de colaboración en el que se negocian significados y se movilizan conocimientos comunes, por lo que se trata pues de entender cómo aplicamos el lenguaje para comprender conjuntamente la experiencia. Además, PérezMateo y Guitert (2007), en su investigación sobre la dimensión social del aprendizaje colaborativo virtual, concluyen que para realizar un verdadero aprendizaje colaborativo, el cual produzca un éxito y satisfacción de todos sus integrantes, debe haber un equilibrio entre los aspectos sociales o afectivos y los cognitivos.

Así, esta construcción compartida de significados a través del lenguaje, como mecanismo interpsicológico del aprendizaje colaborativo, junto con la interdependencia positiva y las relaciones psicosociales, implica: la producción conjunta de objetivos, planes y significados; interpelar y contribuir con explicaciones y argumentaciones;

Mensajería instantánea y construcción compartida de significados: una experiencia de aprendizaje colaborativo en el Prácticum de Maestro de Educación Primaria.

José Manuel Martín Fernández y Carmen Trigueros Cervantes

Página 3 de 18 
mediar y coordinar mutuamente las contribuciones, puntos de vista, críticas y roles en la interacción o exponer reflexiones individuales y colectivas.

\subsection{Aprendizaje colaborativo y construcción compartida de significados en el Prácticum de Maestro de Educación Primaria.}

El Prácticum no debe estar exento de transformaciones y adaptaciones para adecuarse al nuevo modelo de universidad que se está implantando con el proceso de convergencia y desde esta investigación tratamos de contribuir a cambiar nuestras prácticas educativas, de manera que el proceso formativo no se oriente a la mera adquisición de conocimiento, sino al desarrollo y afianzamiento de competencias profesionales. Tomando como referencia la propuesta de Zabalza (2006), que plantea que estas transformaciones, deben buscar: centrar el proceso de aprendizaje en el estudiante, cambiar el papel del profesor hacia una labor de mediación, orientarse hacia un amplio espectro de competencias, mejorar la coordinación teórico-práctica, y hacer uso de las nuevas tecnologías de la información y la comunicación, y generar un aprendizaje más autónomo y autogestionado en el alumnado, en función de nuestros condicionantes y contexto, compatible con las competencias más colaborativas.

Entendemos que el Prácticum debe fomentar en el profesorado (supervisores y tutores) y en el alumnado, procesos de aprendizaje colaborativo y construcción compartida de significados, desde el respeto de la autonomía y el fomento de proyectos de trabajo individuales y grupales relacionados con los intereses de la materia, con el fin último de buscar un cambio de actitud que nos aproxime a la cultura colaborativa y nos aleje de la cultura individualista imperante. Romero y Ortiz (2008), centrándose en el Prácticum de Educación Física, concluyen que el diálogo y la colaboración son herramientas indispensables para allanar el camino hacia la acción formativa de calidad.

El aprendizaje colaborativo mediado por ordenador (CSCL), permitirá que no se contemple al aprendiz como persona individual y aislada, sino en interacción con los otros compañeros, compartiendo objetivos y responsabilidades (Dillenbourg, 1999; Johnson y Johnson, 1999). En este caso concreto que nos ocupa, el contacto virtual, permite a través del intercambio de experiencias y conocimientos que el alumnado en formación aprecie la similitud de sus problemas de prácticas, los desdramatice y se oriente hacia soluciones con referencias entre iguales.

La mensajería instantánea, como recurso didáctico en el ámbito académico, es una valiosa herramienta que permite el tratamiento de aspectos puntuales, la exposición de instrucciones, la resolución puntual de inquietudes y además, admite el intercambio de aportes, acción que puede enriquecer aún más este espacio al contribuir a la generación y fortalecimiento de vínculos académicos entre los participantes, gracias a las posibilidades de encuentros significativos en términos de aprendizaje y reflexión. Estos vínculos por supuesto pueden llegar a facilitar la construcción conjunta de significados y el trabajo colaborativo, así como la conformación de comunidades académicas y de aprendizaje (Ceballos y Sánchez, 2010).

\footnotetext{
Mensajería instantánea y construcción compartida de significados: una experiencia de aprendizaje colaborativo en el Prácticum de Maestro de Educación Primaria. 


\section{Diseño metodológico.}

\subsection{Contexto de la investigación.}

La investigación se realiza en la titulación de Maestro en Educación Física, (actualmente extinguida con la creación de los nuevos grados universitarios), en la asignatura Prácticum II (8 semanas) durante tres cursos académicos en la Facultad de Educación de la Universidad de Granada.

\subsection{Participantes.}

Esta experiencia no afectó a todos los alumnos matriculados en dicha asignatura, sino a diferentes grupos (correspondientes a tres cursos académicos) que por diversos motivos no podían acudir a los seminarios presenciales y fueron autorizados a realizar la supervisión on line. En la tabla 1, podemos ver los participantes en la investigación realizada.

\begin{tabular}{|c|c|c|c|c|}
\hline \multirow{2}{*}{ Grupos } & & \multicolumn{3}{|c|}{ Participantes } \\
\cline { 2 - 5 } & & $\begin{array}{c}\text { Estudiantes } \\
\text { Prácticum } \\
\text { II }\end{array}$ & $\begin{array}{c}\text { Supervisores } \\
\text { Universidad }\end{array}$ & $\begin{array}{c}\text { Tutores } \\
\text { Primaria }\end{array}$ \\
\hline \multicolumn{2}{|c|}{ Grupo A } & 14 & 2 & 1 \\
\hline Grupo B & 20 & 2 & - \\
\hline \multirow{2}{*}{ Grupo C } & 1 & 16 & 1 & 1 \\
\cline { 2 - 5 } & 2 & 17 & 1 & - \\
\cline { 2 - 5 } & 3 & 12 & 1 & 1 \\
\hline
\end{tabular}

Tabla 1. Participantes implicados

\subsection{Metodología.}

La investigación se enmarca en el paradigma interpretativo, puesto que procura generar conocimiento desde una comprensión en profundidad, del significado de los fenómenos sociales, a través de la producción de datos descriptivos desde las propias palabras habladas o escritas- por las personas consultadas desde el propio marco de referencia de quien actúa (Taylor y Bogdan, 1987).La metodología es cualitativa porque posee un carácter descriptivo, relacional e interpretativo, (Strauss y Corbin, 2002) y está "orientada a captar el origen, el proceso y la naturaleza de los significados que brotan de la interacción simbólica entre los individuos" (Ruiz Olabuénaga, 2012:15). Dicha metodología dará respuesta a los diferentes ámbitos de recogida de información e indagación sobre la utilización de la mensajería instantánea como estrategia de aprendizaje colaborativo dentro de un marco de tutorización virtual del Prácticum II de Maestro de Educación Primaria.

Mensajería instantánea y construcción compartida de significados: una experiencia de aprendizaje colaborativo en el Prácticum de Maestro de Educación Primaria. 


\subsection{Técnicas de producción de información.}

En relación a las técnicas cualitativas de producción de datos nos centraremos en el análisis de documentos-. Según Álvarez (2008) esta técnica consiste básicamente en "un rastreo de materiales en formato papel, vídeo, audio, ya sean producidos por los miembros de la comunidad estudiada o por el propio investigado" (p. 8). Por su parte, San Fabián (1992) muestra la gran cantidad de documentos que pueden ser analizados como: periódicos, diarios, artículos, libros de texto, cartas, memorias, autobiografías, historias de vida, historias médicas, publicaciones oficiales, epitafios, casos, folletos políticos, fotografías, videos o películas, etc., a la que añadiremos los documentos pertenecientes a las sesiones de mensajería instantánea objeto de estudio de la investigación mantenidas entre alumnado, tutores y supervisores de los diferentes centros educativos (Tabla 2).

\begin{tabular}{|c|c|c|c|c|c|}
\cline { 4 - 6 } & \multirow{2}{*}{ Grupo A } & \multirow{2}{*}{ Grupo B } & \multicolumn{3}{|c|}{ Grupo C } \\
\cline { 4 - 6 } & & & $\mathbf{1}$ & $\mathbf{2}$ & $\mathbf{3}$ \\
\hline $\begin{array}{c}\text { Sesiones de mensajería } \\
\text { instantánea }\end{array}$ & 4 & 7 & 3 & 3 & 3 \\
\hline
\end{tabular}

Tabla 2. Documentos analizados

El entorno de trabajo se constituyó a través del Messenger que facilita las sesiones de mensajería instantánea, en las que 15 participantes podían intervenir en la misma conversación. Además se hace uso del correo electrónico, así como de la plataforma Swad (Servicio Web de Apoyo a la Docencia, disponible para todo el profesorado y alumnado de la Universidad de Granada).

\subsection{Proceso de análisis de la información.}

Para el análisis de la información nos hemos guiado por Strauss y Corbin (2002) y los pasos que marcan en su Teoría Fundamentada, lo que hace que tengamos que adentrarnos en un proceso de búsqueda caracterizado por el análisis detallado, comprensivo, sistemático y en profundidad de los documentos utilizados. Basándonos en dicha teoría fundamentada se recurre a una perspectiva inductiva, es decir, de lo particular a lo general. Destacar como un recurso de gran ayuda el software Nvivo 10 para el análisis de datos cualitativos (Bazeley y Richards 2000; Bazeley, 2007; Bryman, 2009; Valdemoros, Ponce de León y Sanz, 2011), que nos ha proporcionado un apoyo para efectuar, tanto las tareas de almacenamiento, organización, recuperación,... de todos los archivos y documentos, como las tareas propias del análisis de datos, tales como las búsquedas de texto, agrupación de ideas y temáticas, creación de matrices de intersección y mapas conceptuales, elaboración de teorías, etc.

En una fase inicial de inducción, realizamos una codificación abierta, también llamada codificación 'in vivo'. Como unidad de análisis partimos de las diferentes conversaciones o mensajes que tienen lugar en las sesiones de mensajería instantánea. Utilizando el lenguaje de los participantes, se identifican las ideas, conceptos y significados que contienen una alta significación interpretativa en los documentos (Cuñat, 2007). Después de esta fase inicial, ha sido el momento de la organización de datos desde la identificación de categorías centrales que serán el eje del análisis. Los conceptos y los significados se agrupan en categorías estructuradas por temas y

Mensajería instantánea y construcción compartida de significados: una experiencia de aprendizaje colaborativo en el Prácticum de Maestro de Educación Primaria.

José Manuel Martín Fernández y Carmen Trigueros Cervantes

Página 6 de 18 
metáforas que se encuentran en los textos identificando los principales patrones de análisis.

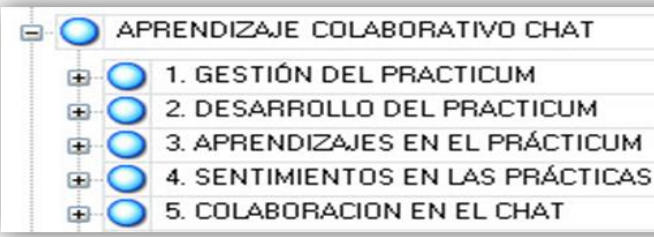

Figura 1. Grandes categorías de indexación o patrones de análisis

Para centrar el análisis en el objeto de estudio de esta investigación combinamos la estrategia inductiva y la deductiva, partiendo de la teoría e investigaciones previas relacionadas con el aprendizaje colaborativo en entornos virtuales, que han precisado y conceptualizado diferentes dimensiones o categorías del aprendizaje colaborativo (Casanova, 2008; García del Dujo y Suárez, 2011; Garrison y Anderson, 2005; Han y Hill, 2007; Johnson y Johnson, 1999; Marcelo y Perera, 2007; Tirado, Hernando y Aguaded, 2011). Así, definimos dentro de la categoría "5. Colaboración en el Chat" un sistema de subcategorías (ver figura 2) que tiene el objetivo de identificar los tres mecanismos interpsicológicos propios de un aprendizaje colaborativo: interdependencia positiva, relaciones psicosociales y construcción de significado; apartado este último que será propósito nuestro de análisis.

\begin{tabular}{|c|c|c|}
\hline \multicolumn{3}{|l|}{ Nodos ramificados } \\
\hline 1 Nombre & Recursos & Referencias \\
\hline 5. COLABORACIÓN EN EL CHAT & 22 & 3110 \\
\hline 5.1. RELACIONES PSICOSOCIALES & 21 & 932 \\
\hline . 5.1.1. Saludos & 17 & 166 \\
\hline 5.1.2. Despedidas & 18 & 190 \\
\hline 5.1.3. Despistes & 15 & 67 \\
\hline 5.1.4. Excusas & 14 & 53 \\
\hline 5.1.5. Agradecimientos & 8 & 27 \\
\hline 5.1.6. Animación & 9 & 23 \\
\hline 5.1.7. Refuerzo-Aprobación & 20 & 296 \\
\hline . 5.1.8. Otros comentarios & 19 & 166 \\
\hline 5.2. INTERDEPENDENCIA POSITIVA & 21 & 1742 \\
\hline † 5.2.1. Gestión del Chat & 19 & 220 \\
\hline 5.2.2. Compartir experiencias & 20 & 1052 \\
\hline 5.2.3. Compartir recursos y otras cosas & 13 & 51 \\
\hline 5.2.4. Interes por los compañeros-Interpela responsabilidad & 20 & 546 \\
\hline 5.2.5. Responsabilidad individual & 10 & 21 \\
\hline$\boxminus O$ 5.3. CONSTRUCCION COMPARTIDA DE SIGNIFICADOS & 21 & 1266 \\
\hline 5.3.1. Cuestiones & 19 & 187 \\
\hline 5.3.2. Aporte de soluciones & 17 & 400 \\
\hline 5.3.3. Explica - Argumenta - Aclara & 20 & 674 \\
\hline 5.3.4. Criticar información - Discrepancias & 19 & 134 \\
\hline
\end{tabular}

Figura 2. Sistema de categorías para el análisis de la "Colaboración en el Chat"

Dentro de la categoría "5.3. Construcción compartida de significados" definimos las siguientes subcategorías:

Mensajería instantánea y construcción compartida de significados: una experiencia de aprendizaje colaborativo en el Prácticum de Maestro de Educación Primaria. 
5.3.1. Cuestiones: aportaciones en modo interrogativo que buscan la aclaración de determinados aspectos.

5.3.2. Aporte de soluciones: aportación de soluciones a los casos planteados en las conversaciones de los chats.

5.3.3. Explica-Argumenta-Aclara: clarificar o hacer comprensible una idea, contenido o concepto expresando el propio punto de vista, a través de opiniones, perspectivas o reflexiones apoyadas en razones.

5.3.4. Criticar información-Discrepancias: valoración crítica de la información aportada por los compañeros con una finalidad constructiva / Manifestaciones de desacuerdo con ideas, contenidos o propuestas para la reflexión y construcción.

\section{Resultados.}

El mecanismo interpsicológico se ha puesto de manifiesto en los seminarios virtuales a través del planteamiento de cuatro aspectos: cuestiones, el aporte de soluciones a problemas surgidos, explicaciones y argumentaciones de los propios puntos de vista ante los diferentes temas emergidos en los chats y la generación de diálogos a partir de discrepancias y valoraciones críticas a informaciones aportadas por los compañeros.

Al analizar la categorización de la construcción compartida de significados en el conjunto de todos los chat (ver tabla 3) se observa que la categoría "5.3.3. ExplicaArgumenta-Aclara" y "5.3.2. Aporte de soluciones" concentran la mayor parte de las intervenciones con 672 y 400 referencias, respectivamente, seguidas de la categoría "5.3.1. Cuestiones" con 187 referencias y "5.3.4. Criticar información-Discrepancias" con 133. Esto nos va a facilitar realizar una primera aproximación a la identificación de las temáticas o intervenciones que principalmente han ocupado los chat, siendo utilizados por los estudiantes para construir conceptos, ideas o significados y llegar a un aprendizaje conjunto a lo largo de los diferentes seminarios chat del Prácticum II.

\begin{tabular}{|l|r|r|r|r|r|r|r|}
\cline { 2 - 7 } 5.3. Construcción compartida de significados & Grupo A & Grupo B & Grupo C1 & Grupo C2 & Grupo C3 & \\
\hline 5.3.1. Cuestiones & 18 & 86 & 37 & 25 & 21 & 187 \\
\hline 5.3.2. Aporte de soluciones & 25 & 46 & 267 & 44 & 18 & 400 \\
\hline 5.3.3. Explica-Argumenta-Aclara & 17 & 161 & 285 & 103 & 106 & 672 \\
\hline 5.3.4. Criticar información-Discrepancias & 14 & 21 & 66 & 16 & 16 & 133 \\
\hline
\end{tabular}

Tabla 3. Distribución de las referencias de la categoría "5.3. Construcción compartida de significados"

\subsection{Argumentos-explicaciones Vs Críticas-discrepancias.}

El elevado número de referencias de la categoría "5.3.3. Explica-Argumenta-Aclara" (672) nos muestra que, coincidiendo con el estudio de Casanova (2008), las intervenciones de los alumnos se han caracterizado por dar explicaciones, aclaraciones y/o argumentos a sus compañeros. Por ejemplo, en la siguiente cita los alumnos explican y argumentan en sus intervenciones la conveniencia sobre su implicación en las actividades y juegos junto con los niños en clase de educación física:

Mensajería instantánea y construcción compartida de significados: una experiencia de aprendizaje colaborativo en el Prácticum de Maestro de Educación Primaria. 
«EMMA DICE ${ }^{1}$ : yo en cuanto puedo me impico en las activiades [...]

ARIEL DICE: está claro q si ellos te ven participar ellos se motivan más porque ven $q$ lo $q$ hacen es weno, nosotros somos como unos pasos a imitar $[\ldots]$

MERLÍN DICE: eso es verdad, al implicarte les motiva a todos los niños que no quieran jugar[...]

BERTA DICE: es muy importante el interés y entusiasmo $k$ le ponga el maestro a la hora de realizar la actividad» (Grupo B, Chat 5b)

Todos estos argumentos y explicaciones que se exponen en los chats, además de formar la categoría con mayor presencia, se convierten en el origen de otras muchas intervenciones de participantes que enriquecen esa construcción de significados en los seminarios. Así aparecen mensajes de refuerzo o aprobación, matizando las afirmaciones, datos o justificaciones, "yo toy de acuerdo con Otto, porque los valores hoy en día en los colegios brillan por su ausencia» (Mateo, Grupo A, Chat 2), o mensajes en los que se manifiestan desacuerdos con las ideas, contenidos o propuestas, valorando críticamente la información aportada por los compañeros. Esto hace emerger la categoría "5.3.4. Criticar información-Discrepancias":

«HÉRCULES DICE: en una clase hice jugar a niñas contra niñas y niños contra niños (2 partidos simultaneos) pq el nivel de habilidad era muy diferente y a ellos les pareció bien, aunque solo fuen para salir del paso $[\ldots]$

ÍCARO DICE: no me parece bien Hércules hay que fomentar la coeducación así como las relaciones interpersonales»(Grupo A, Chat 1).

Con esta estructuración en la que se combinan argumentaciones, explicaciones o discrepancias, observamos como la aplicación de mensajería instantánea facilita la creación de debates colectivos. Debates en los que se tratan diferentes temáticas relacionadas con sus experiencias vividas en las prácticas o concepciones sobre la docencia, la educación, problemas en el aula, etc. de una manera espontánea.

En este sentido, cabe destacar que la codificación de las categorías "5.3.3. ExplicaArgumenta-Aclara" y "5.3.4. Criticar información-Discrepancias" interseccionan con diferente intensidad en el resto de las grandes categorías de análisis (ver tabla 4).

\begin{tabular}{|c|c|c|c|}
\hline & & 5.3.3. Explica - Argumenta - Aclara $\quad \nabla$ & 5.3.4. Criticar información - Discrepancias $\nabla$ \\
\hline 1. GESTIÓN DEL PRÁCTICUM & $\nabla$ & 11 & 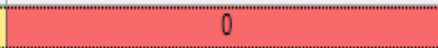 \\
\hline 2. DESARROLLO DEL PRACTICUM & $\nabla$ & 708 & 152 \\
\hline 3. APRENDIZAJES EN EL PRACTICUM & $\nabla$ & 16 & 2 \\
\hline 4. SENTIMIENTOS EN LAS PRACTICAS & $\nabla$ & 6 & 0 \\
\hline
\end{tabular}

\footnotetext{
${ }^{1}$ Las citas de las intervenciones en las sesiones del Messenger son textuales, respetando la forma de expresión y faltas de ortografía cometidas por el alumnado. Los nombres reales se han sustituido por nombres ficticios.
}

Mensajería instantánea y construcción compartida de significados: una experiencia de aprendizaje colaborativo en el Prácticum de Maestro de Educación Primaria.

José Manuel Martín Fernández y Carmen Trigueros Cervantes 
Tabla 4. Intersección de las categorías "5.3.3. Explica-Argumenta-Aclara" y "5.3.4. Criticar información-Discrepancias" con el resto de las grandes categorías de análisis

Así prevalecen las argumentaciones en todas las temáticas, pero si profundizamos en la categoría "2. Desarrollo del prácticum" se aprecia una mayor presencia de las críticas y discrepancias en subcategorías como "2.2.4. Metodología" y "2.2.6. Dificultades". Un ejemplo claro lo tenemos en los debates más extensos que se han generado a partir de los temas emergentes relacionados con la categoría "2.2.4 Metodología", concretamente comentarios sobre estrategias de organización y control; y la aparición de problemas organización y de disciplina graves en las aulas en la categoría "2.2.6. Dificultades" (ver gráfica 1).

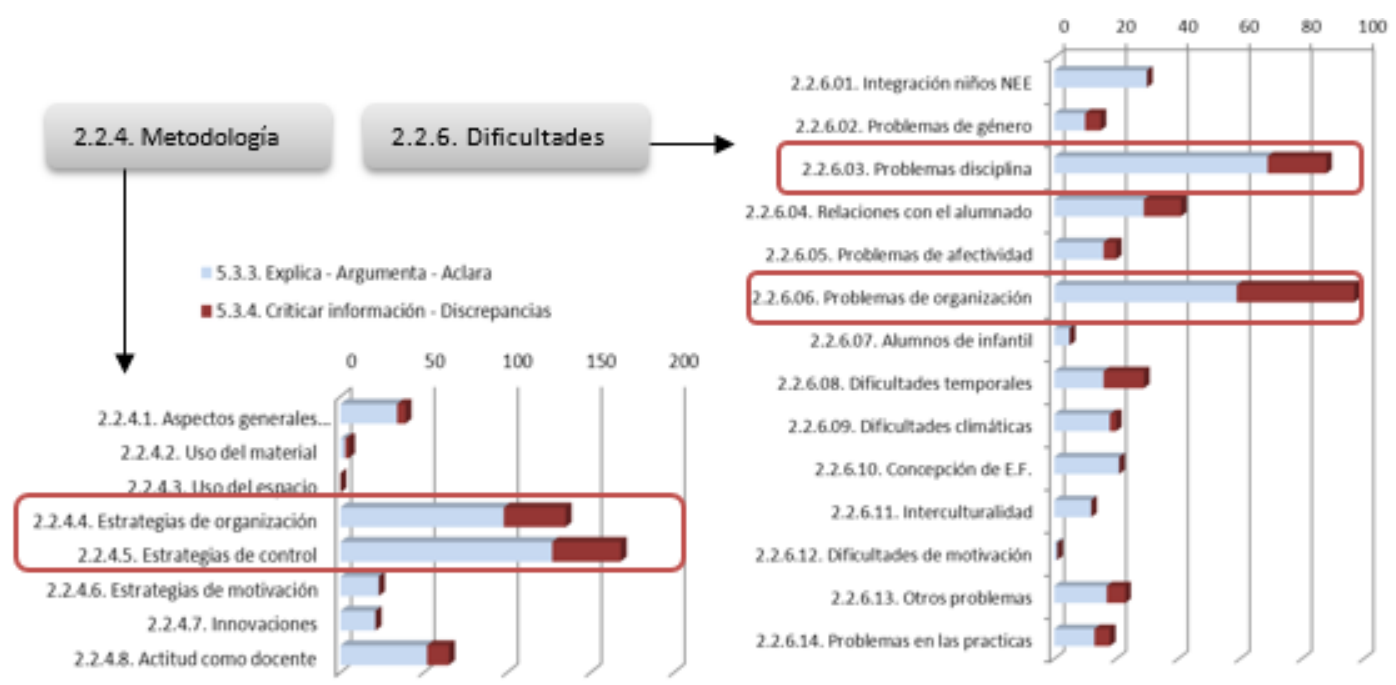

Gráfica 1. Presencia las categorías "5.3.3. Explica-Argumenta-Aclara" y "5.3.4. Criticar información-Discrepancias" en las subcategorías de "2.2.4. Metodología" y "2.2.6. Dificultades".

Si analizamos el foco de debate sobre los líderes que aparece en la subcategoría "2.2.6.06 Problemas de organización”, vislumbramos claramente que para realizar una construcción compartida de significados a partir de la generación de debates en los seminarios chat, su planificación no solo parte del moderador-supervisor, sino que también éstos han surgido en los chat a partir de:

- Iniciativas del alumnado: "...propongo como tema "LOS LIDERES DE LA CLASE"» (Marcos, Grupo C1, Chat 3).

- Intervenciones de los tutores: «...habéis intentado detectar dónde están los alumnos/ alumnas líderes» (Tutor 3, Grupo C2, Chat 2).

- De forma espontánea a raíz de otro hilo de conversación generador: ... «en la clase el tutor lo ha colocado junto al lider (refiriéndose a un alumno inmigrante)»(Norman, Grupo C1, Chat 1).

Mensajería instantánea y construcción compartida de significados: una experiencia de aprendizaje colaborativo en el Prácticum de Maestro de Educación Primaria. 


\subsection{Los aportes de soluciones.}

Uno de los aspectos más significativos que hemos encontrado en el análisis del discurso de las conversaciones surgidas en las sesiones de mensajería instantánea, junto con los extensos debates que han aflorado, son el elevado número de soluciones que aportan los alumnos del Prácticum II cuando se encuentran con diferentes problemas, lo que según Trigueros, Rivera y Torre (2011), facilita el aprendizaje colaborativo de manera innegable.

Indagando cuales son los contextos de enseñanza en la práctica diaria de aula que han provocado una mayor demanda de soluciones, -a partir de realizar una matriz de intersección entre la categoría "5.3.2. Aporte de soluciones" con el resto de categorías surgidas en la investigación-, se observa (ver tabla 5) que éstas se centran fundamentalmente en las subcategorías incluidas en "2.2.4. Metodología" y "2.2.6. Dificultades". Concretamente destacan las intervenciones relacionadas con "2.2.4.5. Estrategias de control", "2.2.4.4. Estrategias de organización", "2.2.6.06 Problemas de organización", "2.2.6.03. Problemas de disciplina" o "2.2.6.01. Integración de alumnos con necesidades educativas especiales".

\begin{tabular}{|lr|c|}
\hline & & 5.3.2. Aporte de soluciones \\
\hline 2.2.4.1. Aspectos generales metodológicos & $\nabla$ & 8 \\
\hline 2.2.4.2. Uso del material & $\nabla$ & 8 \\
\hline 2.2.4.4. Estrategias de organización & $\nabla$ & 54 \\
\hline 2.2.4.5. Estrategias de control & $\nabla$ & 94 \\
\hline 2.2.4.7. Innovaciones & $\nabla$ & 18 \\
\hline 2.2.4.8. Actitud como docente & $\nabla$ & 13 \\
\hline 2.2.6.01. Integración niños NEE & $\nabla$ & 32 \\
\hline 2.2.6.03. Problemas disciplina & $\nabla$ & 42 \\
\hline 2.2.6.04. Relaciones con el alumnado & $\nabla$ & 14 \\
\hline 2.2.6.05. Problemas de afectividad & $\nabla$ & 18 \\
\hline 2.2.6.06. Problemas de organización & $\nabla$ & 45 \\
\hline 2.2.6.07. Alumnos de infantil & $\nabla$ & 13 \\
\hline 2.2.6.09. Dificultades climáticas & $\nabla$ & 9 \\
\hline 2.2.6.13. Otros problemas & $\nabla$ & 10 \\
\hline 2.2.6.14. Problemas en las practicas & $\nabla$ & 27 \\
\hline
\end{tabular}

Tabla 5. Distribución de la categoría "5.3.2. Aporte de soluciones" en las diferentes categorías

A modo de ejemplo, dentro de la categoría "2.2.4.5. Estrategias de control" la preocupación por dichas estrategias se ha centrado en una serie de soluciones en torno a las utilizadas por el profesor para dar la información inicial, "otra opción es quedarte quieto y en silencio mirándolos, pq al cabo de 1 minuto están todos callados...» (Odín, Grupo C2, Chat 2), o los castigos o el diálogo como recurso para solucionar conflictos, «creo que lo mejor es hablar con el niño y hacerle entender por qué su comportamiento está mal y como debe cambiarlo...» (Venus, Grupo A, Chat 1). Mientras que los problemas de organización que se han encontrado los estudiantes han demandado ayuda para realizar los agrupamientos en los diferentes juegos o actividades, "yo he utilizado colores de camisetas» (Emma, Grupo B, Chat 5b).

También observamos en el cruce de categorías de la tabla 5, los problemas de disciplina y la necesidad de integración de alumnos con necesidades educativas especiales en la clase, que han dado origen a gran cantidad de propuestas para solucionar dichas situaciones; como el uso de los tiempos fuera, la realización de tareas alternativas, la

Mensajería instantánea y construcción compartida de significados: una experiencia de aprendizaje colaborativo en el Prácticum de Maestro de Educación Primaria.

José Manuel Martín Fernández y Carmen Trigueros Cervantes 
derivación del alumno disruptivo al director o tutor del grupo, reclamar asesoramiento de personas más cualificadas, etc. Además se produce una menor frecuencia de intervenciones en las que los alumnos aportan su granito de arena para solucionar los problemas de sus compañeros, reflejadas en categorías como: "2.2.4.7. Innovaciones", "2.2.4.8. Actitud como docente", "2.2.6.0. Dificultades climáticas" o "2.2.4.2. Uso de materiales específicos".

Toda esta búsqueda conjunta de soluciones para tratar de resolver los principales problemas y dificultades encontradas en el Practicum II, refleja actitudes de ayuda y solidaridad que emergen en las sesiones de mensajería instantánea entre los estudiantes. Se toma conciencia de las necesidades de los demás expresando un deseo de contribuir y de colaborar para su satisfacción, como podemos observar en la intervención de Lucas cuando empatiza con Selena:

\section{«LUCAS DICE: Selena, poco a poco}

LUCAS DICE: yo por eso siempre intento ponerlo como el ejemplo, MUY BIEN X!!! MUY BIEN!!! MIRAD COMO LO HACE EL!!!, venga vamos a explicarlo los dos juntos...» (Grupo Cl, Chat 1)

\subsection{Planteamiento de cuestiones.}

Por último mencionar que los chats también han sido un lugar donde realizar intervenciones en modo interrogativo que buscan la aclaración de determinados aspectos, considerándolas como otro de los indicadores de una construcción compartida de significados entre los estudiantes. En la gráfica 2 -elaborada a partir de realizar una matriz de intersección entre la categoría "5.3.1. Cuestiones" con el resto de categorías surgidas en la investigación-, mostramos la distribución, en número de referencias, de las cuestiones planteadas en los diferentes chat.

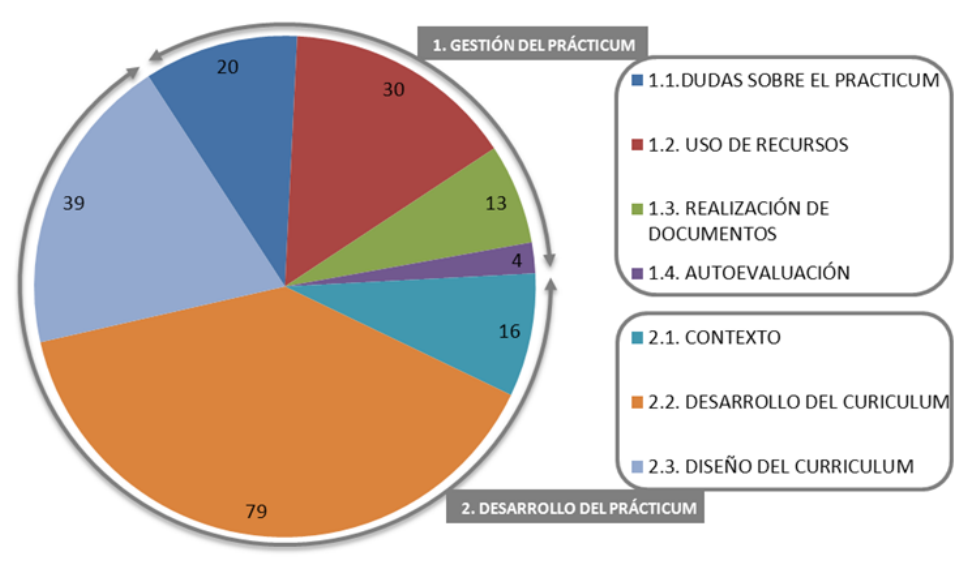

Gráfica 2. Distribución de las referencias de la categoría "5.3.1. Cuestiones"

Las cuestiones planteadas se centran principalmente en el desarrollo del Prácticum II, es decir, en aspectos relacionados con el contexto de la propia intervención educativa en el aula y los elementos necesarios para el diseño y desarrollo del currículum.

Mensajería instantánea y construcción compartida de significados: una experiencia de aprendizaje colaborativo en el Prácticum de Maestro de Educación Primaria. 
A partir de realizar el cruce de la categoría "5.3.1. Cuestiones" con el resto de subcategorías de "2.2. Desarrollo del curriculum" (ver tabla 6), las categorías "2.2.4. Metodología" y "2.2.6. Dificultades" han concentrado la mayor parte de estas cuestiones.

\begin{tabular}{|l|c|c|}
\hline & & 5.3.1. Cuestiones \\
\hline 2.2.1. Aspectos generales & $\nabla$ & 0 \\
\hline 2.2.2. Objetivos y capacidades & $\nabla$ & 5 \\
\hline 2.2.3. Contenidos & $\nabla$ & 13 \\
\hline 2.2.4. Metodología & $\nabla$ & 34 \\
\hline 2.2.5. Evaluación & $\nabla$ & 16 \\
\hline 2.2.6. Dificultades & $\nabla$ & 24 \\
\hline
\end{tabular}

Tabla 6. Intersección de la categoría "5.3.1. Cuestiones" con las subcategorías pertenecientes a "2.2. Desarrollo del curriculum".

Sin embargo también la categoría "2.2.5. Evaluación”, que incluye intervenciones relacionadas con la forma de evaluar al alumnado, así como la categoría "2.2.3. Contenidos", hacen que en algunos chats aparezcan preguntas como:

«IRIS DICE: pero que les entregar fichas para que la hagan??... y que les pones de contenidos??»(Grupo C3, Chat 1)

«JANO DICE: toi un poco perdio, cual es el problema de hacer una prueba escrita?;?[...]»(Grupo 2, Chat 1)

\section{Discusión.}

Casanova (2008), en su investigación sobre el aprendizaje colaborativo en un contexto virtual universitario de comunicación asincrónica, ya presentaba la categoría "explicaargumenta", asociada a la construcción de significado, con el mayor porcentaje $(35,1 \%)$ en relación a las demás categorías. Este autor afirma que este alto porcentaje puede contribuir también a la baja frecuencia de la categoría "discrepa" de su estudio $(3,1 \%)$, pues "al presentarse las ideas más elaboradas, a través de explicaciones y/o argumentaciones, éstas resultan más claras y convincentes para los demás miembros". A pesar de ello, en los debates que se generan en los chat, todos tienen la oportunidad de intervenir contribuyendo con sus argumentos y contra-argumentos de una manera ordenada (Trigueros, Rivera y Torre, 2011). Además, coincidimos con Hernández (2001), en su estudio sobre los usos y potencialidades para fomentar el aprendizaje cooperativo a partir del chat como herramienta de comunicación en la educación a distancia, cuando detectamos cómo estas discusiones o debates grupales han sido una de las estrategias colaborativas para llegar a resolver problemas de forma creativa y realizar tomas de decisiones de forma conjunta. Por su parte, Hyo-jeong y Thomas (2008) también comprobaron que la argumentación en colaboración es una actividad utilizada para fomentar la discusión crítica en ambientes cara a cara y en-línea.

En otros estudios, como los de Tirado, Hernando y Aguaded (2011) y García del Dujo y Suárez (2011), también aparecen los aportes de soluciones y el planteamiento de cuestiones que realizan los estudiantes en foros virtuales de comunicación asincrónica,

Mensajería instantánea y construcción compartida de significados: una experiencia de aprendizaje colaborativo en el Prácticum de Maestro de Educación Primaria.

José Manuel Martín Fernández y Carmen Trigueros Cervantes 
como indicadores o categorías que contribuyen a la construcción de un aprendizaje colaborativo. En esta línea, Sandoval (2008) señala como requisito para que la colaboración se pueda llevar a cabo, que debe existir un clima de confianza para abordar los problemas que surjan sin que nadie se sienta juzgado.

\section{Conclusiones.}

A partir del análisis de los resultados de la investigación, y teniendo en cuenta la casuística de realizar el Prácticum II en Centros Educativos que se encontraban fuera de la provincia de Granada, podemos observar que la virtualización de parte de dicha supervisión a través de sesiones de mensajería instantánea (seminarios virtuales), ha facilitado la comunicación y participación de todos los estudiantes participantes en esta investigación. Aspecto al que creemos ha contribuido la familiaridad con la que los actuales estudiantes utilizan las tecnologías y servicios de mensajería, comenzando a estar inmersos en las nuevas generaciones de nativos tecnológicos o digitales.

Sin embargo, más allá de sólo crear espacios de reunión, esta herramienta de mensajería facilita la desinhibición, haciendo aflorar actitudes colaborativas con el objetivo de generar un conocimiento común o construcción de significados a partir de las experiencias vividas en el Prácticum II. Construcción compartida de significados que se pone de manifiesto a través de los extensos debates generados a partir de los argumentos y discrepancias en las temáticas surgidas, la actitud solidaria de aporte de soluciones a problemas planteados, planteamiento de cuestiones, o reflexiones individuales $\mathrm{y}$ colaborativas.

En este contexto de colaboración, la capacidad para aprender y construir el conocimiento se muestra como el resultado de la habilidad para relacionarnos con otras personas de las que aprendemos y con las que afrontamos multitud de experiencias. Una colaboración para conseguir objetivos comunes, una colaboración para poder hacer frente a los problemas surgidos, una colaboración para compartir nuestras experiencias y conocimientos y beneficiarnos de los ajenos.

Entendemos así, que la utilización de estas herramientas virtuales con potencial para facilitar las relaciones sociales entre las personas permita llevar hasta sus últimas consecuencias procesos colaborativos dentro del Prácticum II de Maestro de Educación Primaria. Un prácticum organizado para que todos sus integrantes se beneficien de la integración de las competencias, conocimientos y experiencias de todas las personas.

Presentación del artículo: 3 de septiembre de 2016 Fecha de aprobación: 19 de octubre de 2016 Fecha de edición: 15 de noviembre de 2016

Martín, J. M. y Trigueros, C. (2016). Mensajería instantánea y construcción compartida de significados: una experiencia de aprendizaje colaborativo en el Prácticum de Maestro de Educación Primaria. RED. Revista de Educación a Distancia, 51. Consultado el (dd/mm/aaaa) en http://www.um.es/ead/red/51/

Mensajería instantánea y construcción compartida de significados: una experiencia de aprendizaje colaborativo en el Prácticum de Maestro de Educación Primaria. 


\section{Referencias bibliográficas.}

Álvarez, C. (2008). La etnografía como modelo de investigación en educación. Gaceta de Antropología, 24(1). Disponible en: http://hdl.handle.net/10481/6998

Bazeley P, y Richards L. (2000). The NVivo Qualitative Project Book. London: Sage Publications.

Bazeley, P. (2007). Qualitative Data Analysis with NVivo. Thousand Oaks, CA: Sage.

Bryman, A. (2009). Computer-assisted qualitative data analysis: using NVivo. En Social Research Methods. Third Edition. Oxford University Press, Oxford.

Calzadilla, E. (2002). Aprendizaje colaborativo y tecnologías de la información y la comunicación. Revista Iberoamericana de Educación, [Apartado de los lectores]. Disponible en: http://www.rieoei.org/deloslectores/322Calzadilla.pdf

Casanova, M. O. (2008). Aprendizaje Cooperativo en un Contexto Virtual Universitario de Comunicación Asincrónica: Un estudio sobre el proceso de interacción entre iguales a través del análisis del discurso [Tesis doctoral]. Universidad Autónoma de Barcelona, Bellaterra. Disponible en: http://hdl.handle.net/10803/4771

Casanova, M.O.; Álvarez, I.M. y Gómez, I. (2009). Propuesta de indicadores para evaluar y promover el aprendizaje cooperativo en un debate virtual. Edutec-e, Revista Electrónica de Tecnología Educativa 28, 1-17. Disponible en http://edutec.rediris.es/Revelec2/revelec28/articulos_n28_pdf/EdutecE_Casanova_Alvarez_Gomez_n28.pdf

Ceballos, L. M. y sánchez, A. (2010). Usos académicos del chat y estrategias lingüísticas en la comunicación virtual sincrónica. Revista Virtual Universidad Católica del Norte, 30, 10-35. Disponible en http://www.redalyc.org/articulo.oa?id=194214476002

Cuadrado, I., Fernández, I., Monroy, F.A., Montaño, A. (2012). Estilos de aprendizaje del alumnado de Psicopedagogía y su implicación en el uso de las TIC y aprendizaje colaborativo. RED. Revista de Educación a Distancia, 35. Disponible en: http://www.um.es/ead/red/35/

Cuñat, R. J. (2007). Aplicación de la teoría fundamentada (grounded theory) al estudio del proceso de creación de empresas. Decisiones basadas en el conocimiento y en el papel social de la empresa. XX Congreso anual de AEDEM 2, 44. Disponible en: http://dialnet.unirioja.es/servlet/ articulo?codigo=2499458

Dillenbourg P. (1999) What do you mean by collaborative leraning?. En P. Dillenbourg (Ed). Collaborative-learning: Cognitive and Computational Approaches. pp. 119. Oxford: Elsevier

Fernández Jiménez, L. (2002). La función y uso del Chat desde los modelos del currículum en la formación del profesorado. Comunicación presentada en el II Congreso Europeo de Tecnología en la Educación y la Ciudadanía: Una visión

Mensajería instantánea y construcción compartida de significados: una experiencia de aprendizaje colaborativo en el Prácticum de Maestro de Educación Primaria.

José Manuel Martín Fernández y Carmen Trigueros Cervantes 
Crítica. Barcelona, 26, 27 y 28 Junio; Disponible en: http://web.udg.edu/tiec/cast/principalnegran.htm

Fernández, M ${ }^{\mathrm{a}}$ D. y Malvar, $\mathrm{M}^{\mathrm{a}}$ L. (1999). La colaboración en los centros educativos: una oportunidad de aprendizaje profesional. Profesorado. Revista de currículum y formación del profesorado, 31. Disponible en: http://www.ugr.es/ recfpro/rev31COL3.pdf

García del Dujo, A. y Suárez, C. (2011). Interacción virtual y aprendizaje cooperativo. Un estudio cualitativo. Revista de Educación, 354, 473-498. Disponible en: http://www.revistaeducacion.mec.es /re354/re354_19.pdf

Garrison, D.R y Anderson, T. (2005). El e-learning en el siglo xxi: Investigación y Práctica. Barcelona: Octaedro

Gros, B. (2007). El aprendizaje colaborativo a través de la red: límites y posibilidades. Disponible en: http://www.uninorte.edu.co/congresog10/conf/08_El_Aprendizaje_Colaborativo a_traves_de_la_red.pdf

Gros, B., Guerra, V. y Sánchez, J. (2005). El aprendizaje colaborativo en la enseñanza superior. Encounters on education, 6, 23-42.

Gutiérrez, P., Yuste, R., Cubo, S. y Lucero, M. (2011). Buenas prácticas en el desarrollo de trabajo colaborativo en materias TIC aplicadas a la educación. Profesorado, Revista de curriculum y formación del profesorado, 151. Disponible en: http://www.ugr.es/local/recfpro/rev151ART13.pdf

Han, S. y Hill, J. (2007). Collaborate to learn, learn to collaborate: examining the roles of context, community and cognition in asynchronous discussion. Educational Computing Research, 36(1), 89-123. Disponible en: http://www.umsl.edu/ wilmarthp/mrpc-web-resources/HANN-COLLABORATE-TO-LEARN-LEARN-TO-COLLABORATE.pdf

Hernández, N. M. (2001). El Chat como herramienta de comunicación en la educación a distancia: usos y potencialidades para fomentar el aprendizaje cooperativo. Docencia Universitaria, 2 (2), 27-39. Disponible en: http://www.ucv.ve/fileadmin/user_upload/sadpro/Documentos/ docencia_vol2_n2_2001/5_art._2Nayesia_Hernandez.pdf

Hyo-jeong, S. y Thomas, A. B. (2008). Student perceptions of collaborative learning, social presence and satisfaction in a blended learning environment: Relationships and critical factors. Computers \& Education, vol. 51 (1), 318-336. Disponible en: doi:10.1016/i.compedu.2007.05.009

Imbernón, F. (2000). Un nuevo Profesorado para una nueva Universidad: ¿conciencia o presión? Revista interuniversitaria de formación del profesorado,38, 37-46.

Johnson, D. y Johnson, R. (1999). Aprender juntos y solos. Buenos Aires: Grupo Editorial Aique.

Mensajería instantánea y construcción compartida de significados: una experiencia de aprendizaje colaborativo en el Prácticum de Maestro de Educación Primaria.

José Manuel Martín Fernández y Carmen Trigueros Cervantes 
Koschmann, T. (1996). Paradigm shifts and instructional technology. En T. KOSCHMANN (Ed.), CSCL: Theory and practice of an emerging paradigm $\mathrm{pp}$. 1-23. Mahwah: Lawrence Erlbaum Associates. Disponible en: http://opensiuc.lib.siu.edu/meded_books

Lopéz Alazid, Mª.P. (2008). Efectos del aprendizaje cooperativo en las habilidades sociales, la educación intercultural y la violencia escolar: un estudio bibliométrico de 1997 a 2007. [Tesis doctoral]. Universidad de Alicante. Disponible en: http://www.cervantesvirtual.com/nd/ark:/59851/bmc15522

Lorente, A. (2006). Cultura docente y organización escolar en los Institutos de Secundaria. Profesorado. Revista de currículum y formación del profesorado, 10, 2. Disponible en: http://www.ugr.es/ recfpro/rev102ART5b.pdf

Marcelo, C. y Perera, V. H. (2007). Comunicación y aprendizaje electrónico: la interacción didáctica en los nuevos espacios virtuales de aprendizaje. Revista de Educación, 343, 381-429. Disponible en: http://www.revistaeducacion.mec.es/re343/re343_17.pdf

Mercer, N. (2001). Spoken language in the classroom. En Mesthrie, Rajend ed. The Concise encyclopedia of sociolinguistics. Encyclopedia of Language and Linguistics (9). Amsterdam, Netherlands: Pergamon, 749-752.

Onrubia, J., Naranjo, M. y Segués, M.T. (2009). Debate y construcción de conocimiento en foros virtuales: la importancia de los motivos de los participantes en la actividad. Cultura y Educación, 21 (3), 275-289

Pérez-Mateo, M. y Guitert, M. (2007). La dimensión social del aprendizaje colaborativo virtual. Red, Revista de Educación a Distancia, 18. Disponible en http://www.um.es/ead/red/18

Pizá, R. I. y Madueño, M. L. (2007). El aprendizaje colaborativo apoyado en medios tecnológicos virtuales. Revista Vasconcelos de Educación, 3 (5), 87-101.

Romero, C. y Ortiz, Ma. M. (2008). Algunas reflexiones y propuestas acerca del prácticum en la especialidad de educación física mediante un grupo de discusión. Contextos educativos, 11, 95-112.

Roselli, N. (2011). Proceso de construcción colaborativa a través del Chat según el tipo de tarea. Revista de Psicología, 29 (1), 3-36. Disponible en: http://revistas.pucp.edu.pe/index.php/psicologia/issue/archive

Ruiz Olabuénaga, J. I. (2012). Metodología de la investigación cualitativa. (5 edición). Bilbao: Universidad de Deusto. Serie Ciencias Sociales.

Sandoval, M. (2008). La colaboración y la formación del profesorado como factores fundamentales para promover una educación $\sin$ exclusiones. Contextos educativos, 11, 149-159.

\footnotetext{
Mensajería instantánea y construcción compartida de significados: una experiencia de aprendizaje colaborativo en el Prácticum de Maestro de Educación Primaria. 
San Fabián, J. L. (1992). Evaluación etnográfica de la educación. En. Blasco B. et al. (1992). Perspectivas en la evaluación del sistema educativo. Oviedo: Departamento de Ciencias de la Educación de la Universidad de Oviedo; 13-53.

Segarra, T. (2006). La semipresencialidad en la universidad: una experiencia de aprendizaje colaborativo con el soporte de las TIC. Comunicación presentada en el $4^{o}$ Congreso Internacional Docencia universitaria e innovación. 5-7 Julio 2006. Barcelona: Universidad Politécnica de Cataluña. Iniciativa Digital Politécnica [Cd-Rom].

Strauss, A. y Corbin, J. (2002). Bases de la investigación cualitativa. Técnicas y procedimientos para desarrollar la teoría fundamentada. Colombia: Editorial Universidad de Antioquia.

Taylor, S. y Bogdan R. (1987). Introducción a los métodos cualitativos de investigación. Barcelona: Paidós.

Tirado, R., Hernando, A. y Aguaded, J. I. (2011). Aprendizaje cooperativo on-line a través de foros en un contexto universitario: un análisis del discurso y de las redes. Estudios sobre educación, vol. 20, 49-71. Disponible en: http://hdl.handle.net/10171/18347

Trigueros, C., Rivera, E. y De La Torre, E. (2011). El Chat como estrategia para fomentar el aprendizaje cooperativo. Una investigación en el Prácticum de Magisterio. Profesorado. Revista de Currículum y Formación de Profesorado, 15(1), 195-210. Disponible en: http://www.redalyc.org/articulo.oa?id=56717469014

Valdemoros, Ma. A., Ponce De León, A. y Sanz, E. (2011). Fundamentos en el manejo del Nvivo 9 como herramienta al servicio de estudios cualitativos. Contextos Educativos, 14, 11-29.

Zabalza, M. A. (2006). El Practicum en la formación universitaria: estado de la cuestión. Revista de Educación, 354, 21-43. Disponible en http://www.revistaeducacion.mec.es/re354/ re354_02.pdf

Mensajería instantánea y construcción compartida de significados: una experiencia de aprendizaje colaborativo en el Prácticum de Maestro de Educación Primaria. 